\title{
Beam Alignment for MIMO-NOMA Millimeter Wave Communication Systems
}

\author{
Wanming $\mathrm{Hao}^{1,2}$, Fuhui Zhou ${ }^{3}$, Zheng $\mathrm{Chu}^{1}$, Pei Xiao ${ }^{1}$, Rahim Tafazolli ${ }^{1}$, Naofal Al-Dhahir ${ }^{4}$ \\ ${ }^{1} 5 \mathrm{G}$ Innovation Centre, Institute of Communication Systems, University of Surrey, Guildford GU2 7XH, U.K. \\ ${ }^{2}$ School of Information Engineering, Zhengzhou University, Zhengzhou, China. \\ ${ }^{3}$ Department of Electrical and Computer Engineering, Utah State University, Utah, USA. \\ ${ }^{4}$ Department of Electrical Engineering, University of Texas, Dallas, USA. \\ E-mail:\{w.hao, zheng.chu, p.xiao,r.tafazolli\}@surrey.ac.uk, zhoufuhui@ieee.org, aldhahir@utdallas.edu
}

\begin{abstract}
Millimeter wave (mmWave) communication is a promising technology in future wireless networks because of its wide bandwidths that can achieve high data rates. However, high beam directionality at the transceiver is needed due to the large path loss at mmWave. Therefore, in this paper, we investigate the beam alignment and power allocation problem in a nonorthogonal multiple access (NOMA) mmWave system. Different from the traditional beam alignment problem, we consider the NOMA scheme during the beam alignment phase when two users are at the same or close angle direction from the base station. Next, we formulate an optimization problem of joint beamwidth selection and power allocation to maximize the sum rate, where the quality of service $(\mathrm{QoS})$ of the users and total power constraints are imposed. Since it is difficult to directly solve the formulated problem, we start by fixing the beamwidth. Next, we transform the power allocation optimization problem into a convex one, and a closed-form solution is derived. In addition, a one-dimensional search algorithm is used to find the optimal beamwidth. Finally, simulation results are conducted to compare the performance of the proposed NOMA-based beam alignment and power allocation scheme with that of the conventional OMA scheme.
\end{abstract}

Index Terms-mmWave, Beam alignment, Power allocation, NOMA

\section{INTRODUCTION}

With the rapid increase of mobile data demands, more available frequency spectrum resources are needed, thus the millimeter wave (mmWave) $(30 \mathrm{GHz}$ to $300 \mathrm{GHz}$ ) frequency band, owing to its wide bandwidth, is envisioned to be an effective scheme to increase the system capacity [1] [2]. However, the deployment and application of the mmWave frequency band in wireless communication is a huge challenge. Different from the Sub-6 GHz band, mmWave suffers from severe propagation loss due to its higher carrier frequency, which reduces the coverage range of the base station (BS) [3]. To overcome this challenge, directional beamforming (BF) can be used for achieving high gain, thus increasing the transmission distance [4] [5]. Moreover, realizing directional $\mathrm{BF}$ at mmWave communication is feasible since the short wavelength at mmWave allows more antenna array elements to be packed to the limited physical space at the transceiver [6] [7]. However, the conventional structure of antenna arrays, where each antenna requires one dedicated radio frequency (RF) chain, is unsuitable for mmWave communications. This is because the power consumption of the RF chain is as high as $250 \mathrm{~mW}$ at mmWave frequency, which is much higher compared to $30 \mathrm{~mW}$ at Sub-6 GHz frequency [8]. To reduce the huge power consumption and hardware complexity, the sparse RF chain structure (where the number of RF chains is much less than the number of antennas) was proposed in [9] [10].

On the other hand, accurate channel estimation is a challenging task in mmWave communications due to the use of large scale antenna arrays. Thus, beam alignment is usually adopted, which does not require the explicit estimation of the channel coefficients [11]. Specifically, the BS and user search all beam pairs from the pre-defined codebook and select the best one to maximize the BF gain and then, the selected beam pair is used for data transmission. However, there exists a tradeoff between the beam alignment and the system throughput. Although the narrow beam can bring a higher directional gain, more time is needed for searching all directions at the beam alignment phase, resulting in less time for data transmission [12]. Therefore, the selection of beamwidth is important during the beam alignment phase. Recently, some papers in literature have investigated this problem. The authors in [13] jointly consider the problems of beamwidth selection and scheduling to maximize the system capacity, and two approximation algorithms are proposed. In [14], the authors develop a novel two-step beam search method for a multi-user system. Reference [15] divides each frame into three continuous parts, including energy harvesting, beam alignment and data transmission, and then a joint optimal energy harvesting ratio and beamwidth selection scheme for maximizing the throughput is proposed. A fair user association, beamwidth selection and power allocation problem for maximizing the worst-case user's throughput is studied in [16]. The authors in [17] develop a beam switching technique that effectively reduces the search scope, which exhibits a much lower complexity and higher performance than the current strategies.

In fact, during the beam alignment phase, the BS may select the same beam pair (including the beamwidth and beam direction) to serve different users when those users have the same or close angle direction from the BS. Moreover, a narrower beam may not be the best choice during the beam alignment phase, thus the selection of a wide beamwidth further increases the probability that different users select the same beam pair. However, in previous works such as [11]-[17], each beam is only used to serve one dedicated user, and 
different users are served by time division multiple access (TDMA) or space division multiple access (SDMA). However, the resource such as time and space may not be fully utilized when different users select the same beam. On the contrary, the nonorthogonal multiple access (NOMA) technique can be used to simultaneously serve those users, thus improving the spectrum efficiency [18] [19]. Although there have been a lot of works on NOMA, the tradeoff between the beam alignment and the system throughput in NOMA has not been considered.

To tackle this problem, in this paper, we study the beam alignment and power allocation problem in a NOMA mmWave system. We first introduce the NOMA scheme during the beam alignment phase. Next, we formulate a joint beamwidth selection and power allocation optimization problem to maximize the sum rate subject to the quality of service $(\mathrm{QoS})$ of users and total power constraints. Due to the intractability of solving the formulated problem, we fix the beamwidth and transform the power allocation optimization problem into a convex one. Moreover, a closed-form solution is derived. Next, a one-dimensional search algorithm is used to find the optimal beamwidth. Simulation results show that the proposed NOMAbased beam alignment and power allocation scheme can obtain a higher sum rate in comparison with the conventional OMA scheme.

The rest of this paper is organized as follows. Section. II introduces the system model and problem formulation. Section. III describes in detail the proposed beamwidth selection and power allocation scheme. Simulation results are provided in Section. IV. We conclude this paper in Section. V.

\section{System Model and Problem Formulation}

We consider a downlink mmWave communication system consisting of one BS with Users 1 and $2^{1}$ as shown in Fig. 1. To reduce the hardware cost and energy consumption, all antennas are connected to one RF chain at BS, Users 1 and 2. In general, as shown in Fig. 2, a time duration $T$ (i.e., one frame) includes two phases: $i$ ) beam alignment and $i i$ ) data transmission. During the first phase, the BS broadcasts beams to users at all directions, and then users feedback the best beam pairs to the BS over the control channel. Next, the BS transmits data to Users 1 and 2 with the selected beam at the second phase. In this paper, we mainly focus on NOMAbased beam alignment and power allocation. We assume that User 1 and 2 have the same or close angle direction from the $\mathrm{BS}^{2}$. In general, the beam alignment includes sector-level and beam-level beam alignment. Without loss of generality, we assume that sector-level alignment has been completed before the beam-level alignment phase, and we only consider the beam-level alignment.

Let $\omega^{t}, \omega^{r}$ and $\theta^{t}, \theta^{r}$ be the sector-level and beam-level beamwidths at BS, Users 1 and 2, respectively. To guarantee the same beam pair between Users 1 and 2, we assume that

\footnotetext{
${ }^{1}$ In this paper, we consider two users for simplicity, but the system analysis and the proposed scheme are also applicable for more users.

${ }^{2}$ The above assumption is reasonable, especially for a dense user area. There must exist two or more users that are at the same or close angle direction from the BS.
}

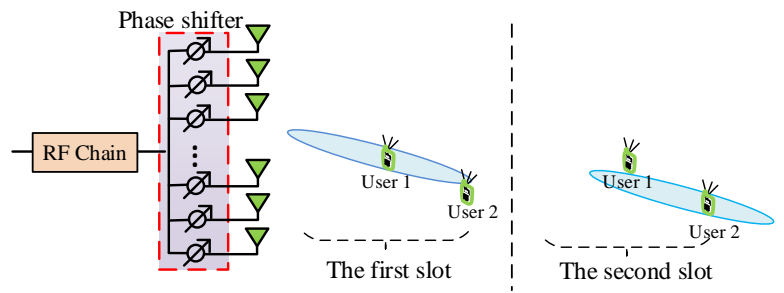

(a)

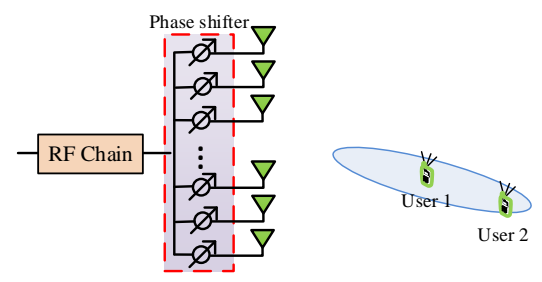

(b)

Fig. 1: (a) The BS serves two users with two narrow beams by TDMA. (b) The BS serves two users with one wide beam by NOMA.

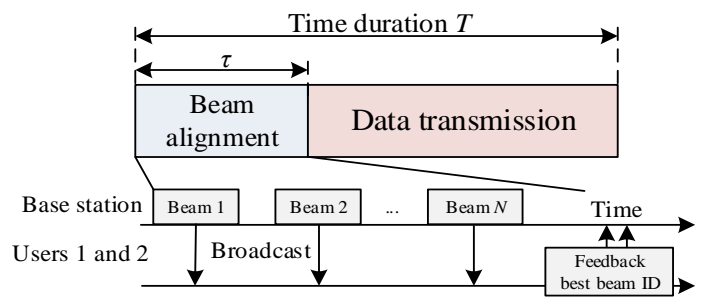

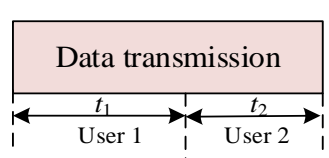

A. The BS serves Users 1 and 2 with TDMA

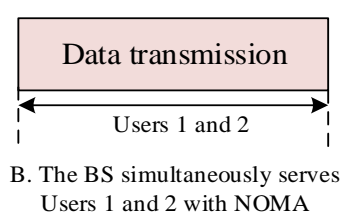

Fig. 2: Beam search and data transmission methods.

$\theta^{t} \geq \theta_{\min }^{r}$ and $\theta^{r} \geq \theta_{\min }^{r}$. When Users 1 and 2 have the same angle direction from the BS, $\theta_{\min }^{t}=\theta_{\min }^{r}=0$. Using the exhaustive search, the total duration time $\tau$ for this searching alignment can be represented as follows

$$
\tau\left(\theta^{t}, \theta^{r}\right)=\left\lceil\frac{\omega^{t}}{\theta^{t}}\right\rceil\left\lceil\frac{\omega^{r}}{\theta^{r}}\right\rceil t_{p}, \quad \theta_{\min }^{t} \leq \theta^{t} \leq \omega^{t}, \theta_{\min }^{r} \leq \theta^{t} \leq \omega^{r},
$$

where $t_{p}$ denotes the pilot transmission time, and $\lceil$.$\rceil is the$ ceiling function.

In this paper, the widely used sectorized antenna pattern model [11]-[17] is adopted, where we assume that the BF gain is equal to a constant for all angles within the main lobe and a small constant within the side lobes. We denote by $\varphi^{t}$ and $\varphi^{r}$ the angles deviating from the strongest path between the BS and user, respectively, as shown in Fig. 3. Then, the transmitting 


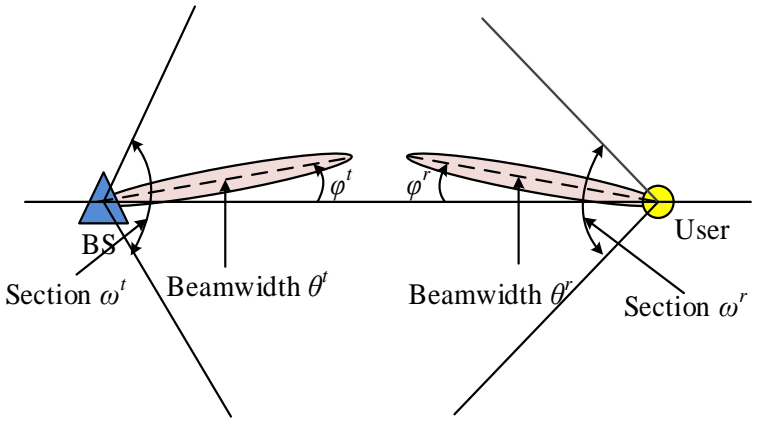

Fig. 3: The analog beamforming alignment procedure.

gain at BS can be expressed as

$$
g^{t}\left(\varphi^{t}, \theta^{t}\right)=\left\{\begin{array}{l}
\frac{2 \pi-\left(2 \pi-\theta^{t}\right) \xi}{\theta^{t}}, \\
\xi, \text { otherwise, }
\end{array}\right.
$$

where $\xi$ is a small constant that represents the side lobe gain. Since Users 1 and 2 have the same or close angle direction from the BS, it is reasonable to assume that they have the same receiving gain as

$$
g^{r}\left(\varphi^{r}, \theta^{r}\right)=\left\{\begin{array}{l}
\frac{2 \pi-\left(2 \pi-\theta^{r}\right) \xi}{\theta^{r}}, \\
\xi, \text { otherwise. }
\end{array}\right.
$$

Once the optimal beam pair is decided by the BS, the beam alignment stage is completed. It is clear that the condition $\left|\varphi^{t}\right| \leq$ $\frac{\theta^{r}}{2}$ in (2) and $\left|\varphi^{r}\right| \leq \frac{\theta^{r}}{2}$ in (3) should be satisfied for the selected beamwidth and BF direction, especially for a wide beam. Next, the BS will transmit data to Users 1 and 2.

Let $h_{1}$ and $h_{2}$ denote the channel gains from the BS to Users 1 and 2, respectively, where we assume $\left|h_{1}\right|>\left|h_{2}\right|$. In addition, $x_{1}$ and $x_{2}$, respectively, represent the transmitted signals for Users 1 and 2. Then, the BS superposes the transmit signals of Users 1 and 2 as

$$
s=\sqrt{P_{1}} x_{1}+\sqrt{P_{2}} x_{2},
$$

where $P_{1}$ and $P_{2}$ denote the transmit powers for Users 1 and 2 , respectively. At the receiver, by using the successive interference cancellation technique [20] [21], User 1 first decodes User 2's signal and then obtains its own signal by subtracting User 2's signal from the received signal. Meanwhile, User 2 directly decodes its own signal by treating User 1's signal as noise. Based on this scheme, the signal to interference plus noise ratio (SINR) of Users 1 and 2 can be expressed as

$$
\begin{aligned}
\gamma_{1} & =\frac{P_{1} h_{1} g^{t}\left(\varphi^{t}, \theta^{t}\right) g^{r}\left(\varphi^{r}, \theta^{r}\right)}{\delta^{2}}, \text { and } \\
\gamma_{2} & =\frac{P_{2} h_{2} g^{t}\left(\varphi^{t}, \theta^{t}\right) g^{r}\left(\varphi^{r}, \theta^{r}\right)}{P_{1} h_{2} g^{t}\left(\varphi^{t}, \theta^{t}\right) g^{r}\left(\varphi^{r}, \theta^{r}\right)+\delta^{2}},
\end{aligned}
$$

respectively, where $\delta^{2}$ denotes the noise power. Hence, Users 1 and 2' rates can be written as

$$
\begin{gathered}
R_{1}\left(\theta^{t}, \theta^{r}, P_{1}\right)=\left(1-\frac{\tau}{T}\right) \log _{2}\left(1+\gamma_{1}\right), \text { and } \\
R_{2}\left(\theta^{t}, \theta^{r}, P_{1}, P_{2}\right)=\left(1-\frac{\tau}{T}\right) \log _{2}\left(1+\gamma_{2}\right),
\end{gathered}
$$

where the transmission time is normalized by time duration $T$.

From (5) and (6), we can observe that a narrower beam leads to a higher SINR. However, according to (1), the beam alignment time $t$ will increase, and the remaining time $T-\tau$ used for data transmission will be decreased. On the other hand, one wide beamwidth can serve Users 1 and 2 more efficiently, especially when they are located in the same angle direction from the BS. Therefore, there exists a tradeoff between the beamwidth and the transmission rate. In this paper, we aim to jointly optimize beamwidth and power allocation for maximizing the sum rate, which can be formulated as

$$
\begin{array}{ll}
\max _{\left\{\theta^{r}, \theta^{r}, P_{1}, P_{2}\right\}} R_{1}\left(\theta^{t}, \theta^{r}, P_{1}\right)+R_{2}\left(\theta^{t}, \theta^{r}, P_{1}, P_{2}\right) \\
\text { s.t. } & R_{1}\left(\theta^{t}, \theta^{r}, P_{1}\right) \geq R_{1}^{\min }, \\
& R_{2}\left(\theta^{t}, \theta^{r}, P_{1}, P_{2}\right) \geq R_{2}^{\min }, \\
& P_{1}+P_{2} \leq P_{\max }, \\
& \theta_{\min }^{t} \leq \theta^{t} \leq \omega^{t}, \\
& \theta_{\min }^{r} \leq \theta^{r} \leq \omega^{r}, \\
& \tau \leq T,
\end{array}
$$

where Equations (9b) and (9c) denote the minimum rate requirement for Users 1 and 2, respectively, and (9d) is the total transmit power constraint for the BS. For (9), we need to find the optimal beam pair $\theta^{t}, \theta^{r}$ and transmit powers $P_{1}, P_{2}$ to maximize the sum rate. In the next section, we propose an effective algorithm to solve the maximization problem.

\section{Proposed Beamwidth Selection and Power Allocation Algorithm}

After completing the beam alignment, the BS and user operate in their main lobes, hence it means $\varphi^{t}=\varphi^{r}=0$. The transmitting and receiving gain can thus be simplified as

$$
g^{t}\left(\theta^{t}\right)=\frac{2 \pi-\left(2 \pi-\theta^{t}\right) \xi}{\theta^{t}}, g^{r}\left(\theta^{r}\right)=\frac{2 \pi-\left(2 \pi-\theta^{r}\right) \xi}{\theta^{r}} .
$$

Next, we define function $G\left(\theta^{t}, \theta^{r}\right)$ as

$$
G\left(\theta^{t}, \theta^{r}\right)=g^{t}\left(\theta^{t}\right) g^{t}\left(\theta^{r}\right)=\frac{\left(2 \pi-\left(2 \pi-\theta^{t}\right) \xi\right)\left(2 \pi-\left(2 \pi-\theta^{r}\right) \xi\right)}{\theta^{t} \theta^{r}} .
$$

Similar to [13] [15], we define $\theta=\theta^{t} \theta^{r}$ and $G\left(\theta^{t}, \theta^{r}\right)$ can be rewritten as

$$
\begin{aligned}
& G\left(\theta^{t}, \theta^{r}\right)=\frac{(2 \pi-2 \pi \xi)^{2}}{\theta}+\frac{(2 \pi-2 \pi \xi) \xi\left(\theta^{t}+\theta^{r}\right)}{\theta}+\xi^{2} . \\
& \Rightarrow G(\theta) \approx \frac{(2 \pi-2 \pi \xi)^{2}}{\theta}+\xi^{2} .
\end{aligned}
$$

The second step means that

$$
\frac{(2 \pi-2 \pi \xi)^{2}}{\theta} \gg \frac{(2 \pi-2 \pi \xi) \xi\left(\theta^{t}+\theta^{r}\right)}{\theta} .
$$

In fact, for a small $\xi$ (i.e., $\xi \ll 1$ ), (13) can be satisfied. This is because (13) implies that

$$
\left(\theta^{t}+\theta^{r}\right) \ll 2 \pi \frac{1-\xi}{\xi},
$$


where we assume that $\xi=0.05$, and $2 \pi \frac{1-\xi}{\xi}=38 \pi \gg\left(\theta^{t}+\theta^{r}\right)$. Therefore, we can approximate $G\left(\theta^{t}, \theta^{r}\right)$ as

$$
G(\theta) \approx \frac{(2 \pi-2 \pi \xi)^{2}}{\theta}+\xi^{2} .
$$

Hence, Users 1 and 2' SINR can be expressed as

$$
\gamma_{1}=\frac{P_{1} h_{1} G(\theta)}{\delta^{2}}, \quad \gamma_{2}=\frac{P_{2} h_{2} G(\theta)}{P_{1} h_{2} G(\theta)+\delta^{2}},
$$

respectively. According to the above analysis, we can reform (9) as the following optimization problem

$$
\begin{array}{ll}
\max _{\left\{\theta, P_{1}, P_{2}\right\}} R_{1}\left(\theta, P_{1}\right)+R_{2}\left(\theta, P_{1}, P_{2}\right) \\
\text { s.t. } & R_{1}\left(\theta, P_{1}\right) \geq R_{1}^{\min }, \\
& R_{2}\left(\theta, P_{1}, P_{2}\right) \geq R_{2}^{\min }, \\
& \theta_{\min }^{t} \theta_{\min }^{r} \leq \theta \leq \omega^{t} \omega^{r}, \\
& (9 \mathrm{~d}),(9 \mathrm{~g}) .
\end{array}
$$

It is easy to observe that (17) is a non-convex optimization problem, which is difficult to solve. In fact, since $\theta \in$ $\left[\theta_{\min }^{t} \theta_{\min }^{r}, \omega^{t} \omega^{r}\right]$, we can adopt the one-dimensional search to find optimal $\theta^{3}$. For a given $\theta=\theta^{\prime}$, we only need to optimize the power levels $P_{1}$ and $P_{2}$ in (17), which can be expressed as

$$
\begin{array}{ll}
\max _{\left\{P_{1}, P_{2}\right\}} R_{1}\left(P_{1}\right)+R_{2}\left(P_{1}, P_{2}\right) \\
\text { s.t. } & R_{1}\left(P_{1}\right) \geq R_{1}^{\min } \\
& R_{2}\left(P_{1}, P_{2}\right) \geq R_{2}^{\min },
\end{array}
$$

(9d).

However, (18) is still difficult to solve in its original form. Next, we will transform (18) into a convex optimization problem by our proposed scheme. According to (16), we have

$$
\begin{array}{r}
P_{1}\left(R_{1}\right)=\frac{2^{R_{1} /(1-\tau / T)}-1}{\beta_{1}}, \text { and } \\
P_{2}\left(R_{2}, P_{1}\right)=\left(2^{R_{2} /(1-\tau / T)}-1\right)\left(P_{1}+\frac{1}{\beta_{2}}\right),
\end{array}
$$

where $\beta_{1}=h_{1} G\left(\theta^{\prime}\right) / \delta^{2}$ and $\beta_{2}=h_{2} G\left(\theta^{\prime}\right) / \delta^{2}$. Substituting (19) into (20), we can obtain

$$
\begin{aligned}
P_{2}\left(R_{1}, R_{2}\right) & =\frac{2^{\left(R_{1}+R_{2}\right) /(1-\tau / T)}}{\beta_{1}}+\left(\frac{1}{\beta_{2}}-\frac{1}{\beta_{1}}\right)\left(2^{R_{2} /(1-\tau / T)}-1\right) \\
& -\frac{2^{R_{1} /(1-\tau / T)}}{\beta_{1}},
\end{aligned}
$$

where $\frac{1}{\beta_{2}}-\frac{1}{\beta_{1}}>0$. Meanwhile, we have

$$
\begin{aligned}
P_{1}\left(R_{1}\right)+P_{1}\left(R_{1}, R_{2}\right) & =\frac{2^{\left(R_{1}+R_{2}\right) /(1-\tau / T)}}{\beta_{1}} \\
& +2^{R_{2} /(1-\tau / T)}\left(\frac{1}{\beta_{2}}-\frac{1}{\beta_{1}}\right)-\frac{1}{\beta_{2}} .
\end{aligned}
$$

\footnotetext{
${ }^{3}$ In fact, $\theta^{t}$ and $\theta^{r}$ should be discrete due to the practical restriction by the antenna parameters. Therefore, in practice, we can select the $\theta^{t}$ and $\theta^{r}$ pair whose product is the closet to the optimal beamwithd $\theta$.
}

Due to the difficulty in optimizing $P_{1}$ and $P_{2}$, we equivalently transform (18) as an optimization problem in $R_{1}$ and $R_{2}$ as follows

$$
\begin{array}{ll}
\max _{\left\{R_{1}, R_{2}\right\}} R_{1}+R_{2} \\
\text { s.t. } & R_{1} \geq R_{1}^{\mathrm{min}}, \quad R_{2} \geq R_{2}^{\min }, \\
& \frac{2^{\left(R_{1}+R_{2}\right) /(1-\tau / T)}}{\beta_{1}}+2^{R_{2} /(1-\tau / T)}\left(\frac{1}{\beta_{2}}-\frac{1}{\beta_{1}}\right)-\frac{1}{\beta_{2}} \leq P_{\max } .
\end{array}
$$

It is obvious that (23) is a convex optimization problem, which can be solved by existing convex solvers (e.g., CVX). In addition, to derive a closed-form solution for the problem (23), a subgradient iterative method can also be utilized [22]. Specifically, we first write the Lagrange function as follows

$$
\begin{aligned}
\mathcal{L} & =R_{1}+R_{2}+\lambda_{1}\left(R_{1}-R_{1}^{\min }\right)+\lambda_{2}\left(R_{2}-R_{2}^{\min }\right) \\
& +\mu\left(P_{\max }-\frac{2^{\left(R_{1}+R_{2}\right) /(1-\tau / T)}}{\beta_{1}}-2^{R_{2} /(1-\tau / T)}\left(\frac{1}{\beta_{2}}-\frac{1}{\beta_{1}}\right)-\frac{1}{\beta_{2}}\right),
\end{aligned}
$$

where $\lambda_{1}, \lambda_{2}$ and $\mu$ are the non-negative dual variables corresponding to constraints (23b) and (23c), respectively. According to the Karush-Kuhn-Tucker (KKT) optimality conditions, we have

$$
\begin{aligned}
\frac{\partial \mathcal{L}}{\partial R_{1}}=1 & +\lambda_{1}-\mu \frac{2^{\left(R_{1}+R_{2}\right) /(1-\tau / T)}}{\beta_{1}(1-\tau / T)} \ln 2, \\
\frac{\partial \mathcal{L}}{\partial R_{2}}=1 & +\lambda_{2}-\mu \frac{2^{\left(R_{1}+R_{2}\right) /(1-\tau / T)}}{\beta_{1}(1-\tau / T)} \ln 2 \\
& -\frac{2^{R_{2} /(1-\tau / T)} \ln 2}{1-\tau / T}\left(\frac{1}{\beta_{2}}-\frac{1}{\beta_{1}}\right) .
\end{aligned}
$$

Combining (25) and (26), we can obtain the optimal $R_{1}$ and $R_{2}$ as

$$
R_{2}=\left(1-\frac{\tau}{T}\right) \log 2\left(\max \left\{\frac{\lambda_{2}-\lambda_{1}}{\mu c}, 1\right\}\right),
$$

where $c=\left(\frac{1}{\beta_{2}}-\frac{1}{\beta_{1}}\right) \frac{\ln 2}{1-\tau / T}$, and

$$
R_{1}=\left(1-\frac{\tau}{T}\right) \log 2\left(\max \left\{\frac{\left(1+\lambda_{1}\right)\left(\beta_{1} / \beta_{2}-1\right)}{\lambda_{2}-\lambda_{1}}, 1\right\}\right) .
$$

The dual variables can be updated with the subgradient method as follows

$$
\begin{aligned}
\lambda_{1}^{(n+1)} & =\left[\lambda_{1}^{(n)}-\alpha_{1}^{(n)}\left(R_{1}-R_{1}^{\min }\right)\right]^{+}, \\
\lambda_{2}^{(n+1)} & =\left[\lambda_{2}^{(n)}-\alpha_{2}^{(n)}\left(R_{2}-R_{2}^{\min }\right)\right]^{+}, \\
\mu^{(n+1)} & =\left[\mu^{(n)}-\alpha_{3}^{(n)}\left(P_{\max }-A_{1}-A_{2}-\frac{1}{\beta_{2}}\right)\right]^{+},
\end{aligned}
$$

where $A_{1}=\frac{2^{\left(R_{1}+R_{2}\right) /(1-\tau / T)}}{\beta_{1}}$ and $A_{2}=2^{R_{2} /(1-\tau / T)}\left(\frac{1}{\beta_{2}}-\frac{1}{\beta_{1}}\right)$, and $\alpha_{i}^{(n)}(i \in\{1,2,3\})$ denotes the step sizes at the $n$th iteration. For the subgradient iterative method, the optimal rates are obtained by (27) and (28). Then, the dual variables are updated according to (29) and the process continues till the iterates converge. After obtaining the optimal $R_{1}$ and $R_{2}$, the optimal power allocation $P_{1}$ and $P_{2}$ can be determined by (19) and (20), respectively. 


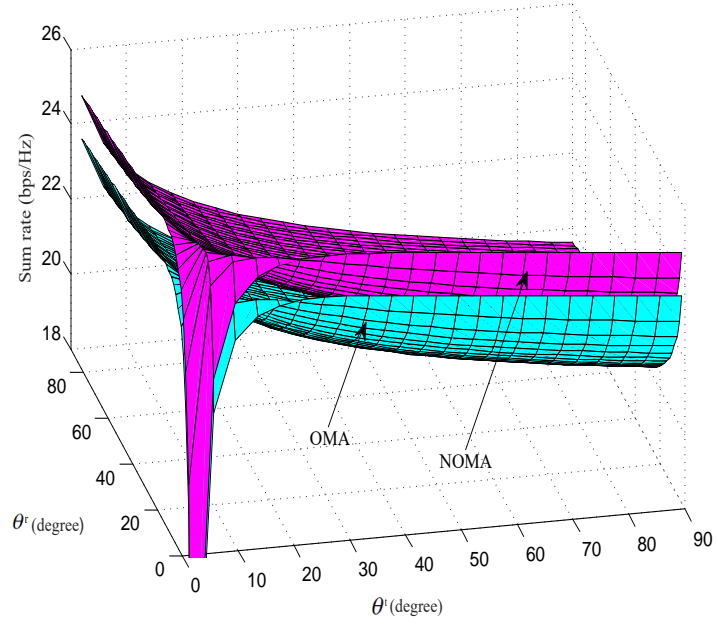

Fig. 4: Sum rate versus beamwidth.

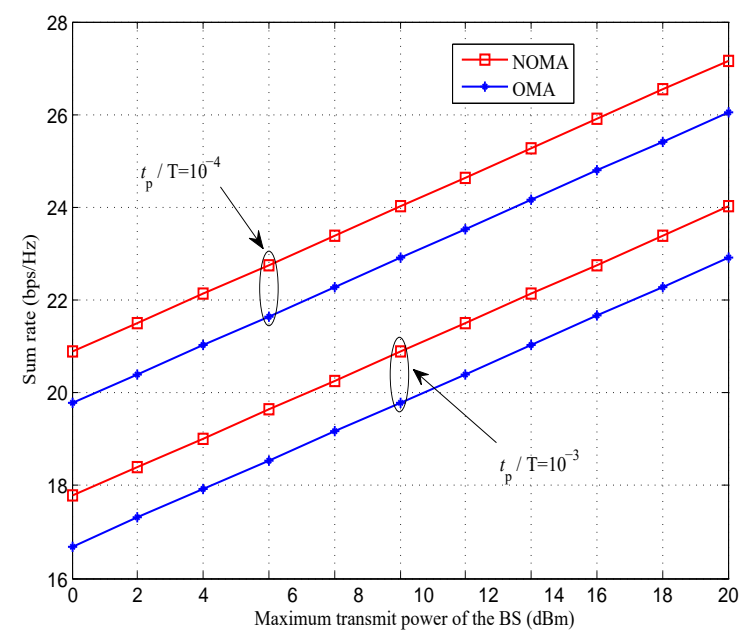

Fig. 5: Sum rate versus maximum transmit power of the BS.

\section{Simulation Results}

In this section, simulation results are presented to demonstrate the effectiveness of the proposed scheme. In addition, as a performance benchmark, we also study an orthogonal multiple access (OMA) scheme, e.g. TDMA, where equal time slots are allocated to Users 1 and 2. The time is normalized to unity, and we assume that the time of pilot transmission is 1, and the total time durations are 1000, 3000 and 10000, respectively, for comparison. The distances between Users 1, 2 and $\mathrm{BS}$ are 20 and 30 meters, respectively. We set the sectorlevel beams $\omega^{t}=\omega^{r}=90^{\circ}$. The path loss is modeled as $69.4+24 \log _{10}(d) \mathrm{dB}$, where $d$ denotes the distance in meters. The noise power $\delta^{2}$ is set as $-80 \mathrm{dBm}$, and the minimum rate is set as $R_{1}^{\mathrm{min}}=R_{2}^{\mathrm{min}}=3 \mathrm{bps} / \mathrm{Hz}$. Other related parameters will be described below.

Fig. 4 shows the sum rate versus beamwidth. We set $P_{\max }=$ $20 \mathrm{dBm}, T=3000, \theta_{\min }^{t}=\theta_{\min }^{r}=0$. It can be observed that although a narrower beam, including $\theta^{t}$ and $\theta^{r}$, has a higher

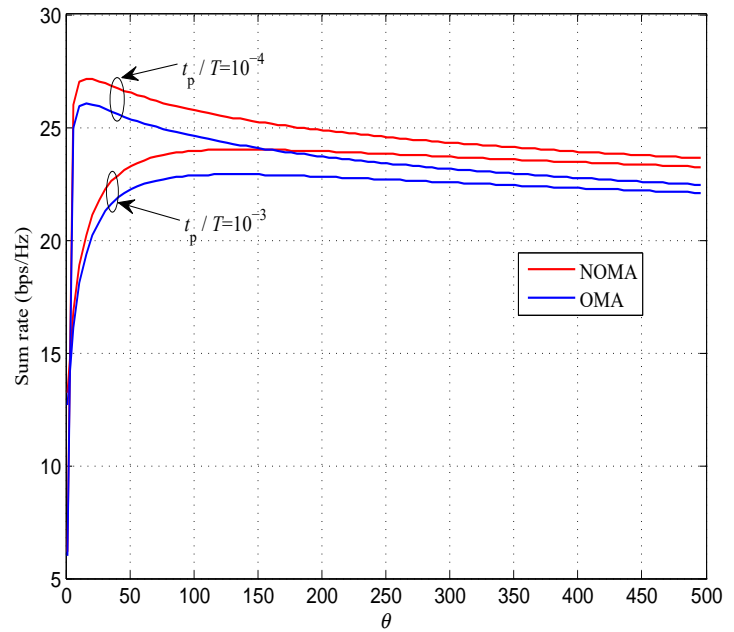

Fig. 6: Sum rate versus $\theta$.

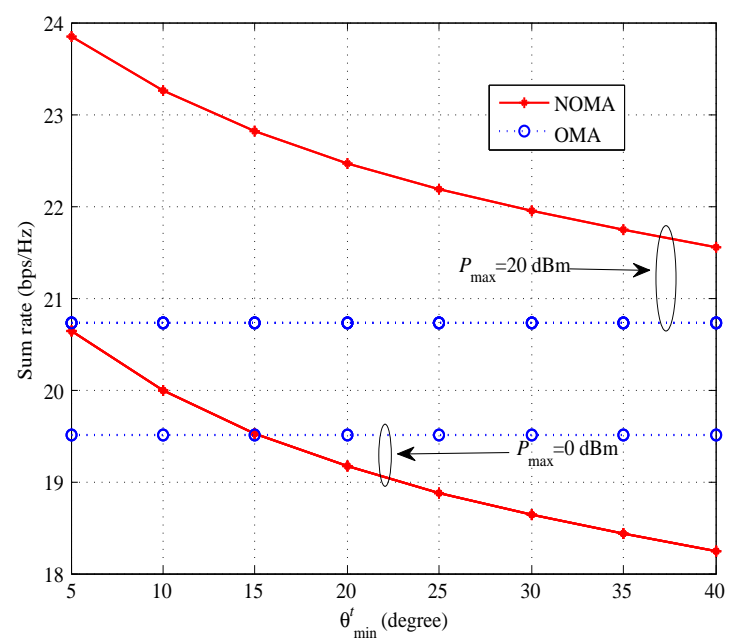

Fig. 7: Sum rate versus $\theta_{\text {min }}^{t}$ with $\theta_{\text {min }}^{r}=5^{\circ}$ and $t_{\mathrm{p}} / T=10^{-4}$.

BF gain, the sum rate turns out to be low. This is because a huge overhead is needed for beam alignment, leaving less time for data transmission. On the other hand, a wider beam is not the optimal selection due to the low BF gain at the transceiver. In addition, one can see that the sum rate under our proposed NOMA scheme is higher than that under the conventional OMA scheme.

Fig. 5 shows the sum rate versus the maximum transmit power of the BS. We set the time duration $T=1000\left(t_{p} / T=\right.$ $\left.10^{-3}\right), T=10000\left(t_{p} / T=10^{-4}\right)$, and $\theta_{\min }^{t}=\theta_{\min }^{r}=0$. One can observe that the sum rate increases with the maximum transmit power. Moreover, it is clear that the longer time duration can bring a higher sum rate due to a longer data transmission time. The NOMA scheme still performs better than the OMA scheme. Meanwhile, Fig. 6 clearly shows that a longer time duration can achieve higher sum rate. Furthermore, we can also observe that the narrow beam does not result in a higher sum rate.

Fig. 7 shows the sum rate versus beamwidth $\theta^{t}$. We set 


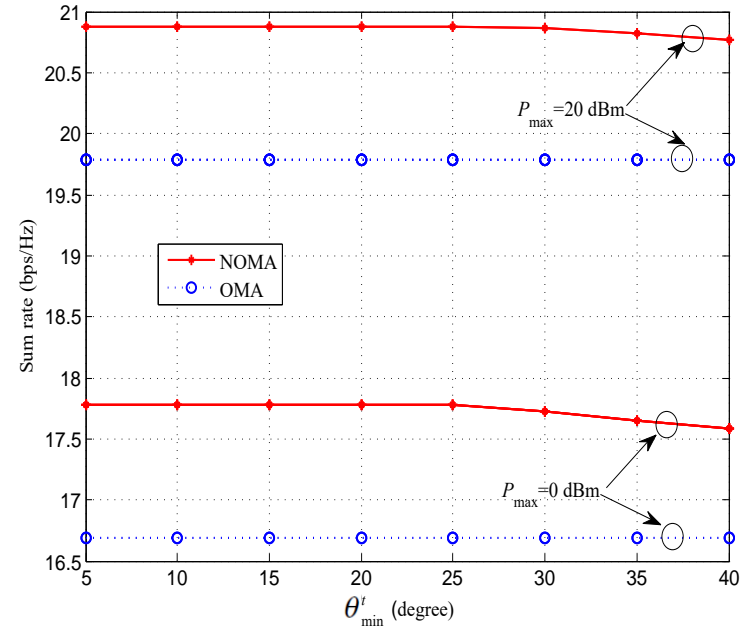

Fig. 8: Sum rate versus $\theta_{\min }^{t}$ with $\theta_{\min }^{r}=5^{\circ}$ and $t_{\mathrm{p}} / T=10^{-3}$.

$\theta_{\min }^{r}=5^{\circ}$ and $t_{\mathrm{p}} / T=10^{-4}$. Note that there is no minimum beamwidth constraint for the OMA scheme, thus the sum rate is a constant. We find that the sum rate decreases with $\theta_{\min }^{t}$. It is easy to understand that a much wider beamwidth results in a lower BF gain, thus decreasing the sum rate. In addition, for $P_{\max }=20 \mathrm{dBm}$, the sum rate under the NOMA scheme is always higher than that under the OMA scheme between $\theta_{\text {min }}^{t} \in\left[5^{\circ}, 40^{\circ}\right]$. However, for $P_{\max }=0 \mathrm{dBm}$, the sum rate under the NOMA scheme is lower than that under the OMA scheme when $\theta_{\min }^{t} \geq 15^{\circ}$. In addition, we set $t_{\mathrm{p}} / T=10^{-3}$ in Fig. 8 , and the other parameters remain the same as in Fig. 7. One can clearly observe that the sum rate under the NOMA scheme is always higher than that under the OMA scheme. The reason can be found in Fig. 6 , the optimal beamwidth with $t_{\mathrm{p}} / T=10^{-3}$ is wider than that with $t_{\mathrm{p}} / T=10^{-4}$. Furthermore, the decrease of the sum rate as $\theta$ with $t_{\mathrm{p}} / T=10^{-3}$ is slower than that with $t_{\mathrm{p}} / T=10^{-4}$ when the sum rate reaches the largest. Overall, the NOMA scheme can achieve a better performance than the OMA scheme, even for a wide beamwidth.

\section{Conclusions}

In this paper, we investigated the beam alignment and power allocation optimization problem to maximize the sum rate in a NOMA mmWave communication system. We proposed to employ the NOMA scheme during the beam alignment phase. In addition, the power allocation optimization problem is transformed into a convex one, and a closed-form solution is obtained. Simulation results showed that the proposed NOMAbased beam alignment and power allocation scheme can achieve a higher sum rate than that of the conventional OMA scheme.

\section{AcKNowledgment}

This work is supported in part by the European Commission under the 5GPPP project 5GXcast (H2020-ICT-20162 call, grant number 761498), and in part by the U.K. Engineering and Physical Sciences Research Council under Grant EP/P008402/1, and in part by the National Natural Science Foundation of China (Grant:61701214), and in part by the Excellent Youth Foundation of Jiangxi Province (Grant 2018ACB21012), and in part by the China Postdoctoral Science Foundation (First rank) (Grant: 2017M610400). The work of N. Al-Dhahir was made possible by NPRP grant number 8627-2-260 from the Qatar National Research Fund (a member of Qatar Foundation). The statements made herein are solely the responsibility of the authors.

\section{REFERENCES}

[1] J. G. Andrews et al., "What will 5G be?" IEEE J. Sel. Areas Commun., vol. 32, no. 6, pp. 1065-1082, Jun. 2014.

[2] W. Hao and S. Yang, "Small cell cluster-based resource allocation for wireless backhaul in two-tier heterogeneous networks with massive MIMO," IEEE Trans. Veh. Technol., vol. 67, no. 1, pp. 509-523, Jan. 2018.

[3] C. Liu, M. Li, S. V. Hanly, I. B. Collings and P. Whiting, "Millimeter wave beam alignment: Large deviations analysis and design insights," IEEE J. Sel. Areas Commun., vol. 35, no. 7, pp. 1619-1631, Jul. 2017.

[4] W. Hao, O. Muta and H. Gacanin, "Price-based resource allocation in massive MIMO H-CRANs with limited fronthaul capacity," IEEE Trans. Wireless Commun., vol. 17, no. 11, pp. 7691-7703, Nov. 2018.

[5] S. Kutty and D. Sen, "Beamforming for millimeter wave communications: An inclusive survey," IEEE Commun. Surveys Tuts., vol. 18, no. 2, pp. 949-973, 2nd Quart., 2016.

[6] X. Gao et al, "Low RF-complexity technologies to enable millimeterwave MIMO with large antenna array for $5 \mathrm{G}$ wireless communications," IEEE Commun. Mag., vol. 56, no. 4, pp. 211-217, Apr. 2018.

[7] W. Hao, M. Zeng, Z. Chu and S. Yang, "Energy-efficient power allocation in millimeter wave massive MIMO with non-orthogonal multiple access," IEEE Wireless Commun. Lett., vol. 6, no. 6, pp. 782-785, Dec. 2017.

[8] R. W. Heath et al., "An overview of signal processing techniques for millimeter wave MIMO systems," IEEE J. Sel. Top. Signal Process., vol. 10, no. 3, pp. 43653, Apr. 2016.

[9] M. Molu et al., "Low-complexity and robust hybrid beamforming design for multi-antenna communication systems. IEEE Trans. Wireless Commun., vol. 17, no. 3, pp. 1445-1459, Mar. 2018.

[10] W. Hao, M. Zeng, Z. Chu, S. Yang and G. Sun, "Energy-efficient resource allocation for mmWave massive MIMO HetNets with wireless backhaul," IEEE Access, vol. 6, pp. 2457-2471, 2018.

[11] Q. Xue, X. Fang and C. Wang, "Beamspace SU-MIMO for future millimeter wave wireless communications," IEEE J. Sel. Areas Commun., vol. 35, no. 7, pp. 1564-1575, Jul. 2017.

[12] D. De Donno, J. Palacios and J. Widmer, "Millimeter-wave beam alignment acceleration through low-complexity hybrid transceivers," IEEE Trans. Wireless Commun., vol. 16, no. 6, pp. 3646-3660, Jun. 2017.

[13] H. Shokri-Ghadikolaei, L. Gkatzikis and C. Fischione, "Beam-searching and transmission scheduling in millimeter wave communications," 2015 IEEE ICC, London, 2015, pp. 1292-1297.

[14] C. Kojima et al., "Novel two-step beam search method for multi user millimeter-wave communication," 2017 IEEE PIMRC, Montreal, QC, 2017, pp. 1-6.

[15] Y. Wu et al.,"Optimal energy harvesting-ratio and beamwidth selection in millimeter wave communications," IEEE Signal Processing Lett., vol. 23, no. 10, pp. 1364-1368, Oct. 2016.

[16] N. Eshraghi, V. Shah-Mansouri and B. Maham, "Fair beamwidth selection and resource allocation for indoor millimeter-wave networks," 2017 IEEE ICC, Paris, 2017, pp. 1-6.

[17] B. Li, Z. Zhou, W. Zou, X. Sun and G. Du, "On the efficient beamforming alignment for $60 \mathrm{GHz}$ wireless personal area networks," IEEE Trans. Wireless Commun., vol. 12, no. 2, pp. 504-515, Feb. 2013

[18] W. Hao et al., "Green communication for NOMA-based CRAN," IEEE Internet of Things Journal, vol. 6, no. 1, pp. 666-678, Feb. 2019.

[19] S. M. R. Islam, M. Zeng, O. A. Dobre and K. Kwak, "Resource allocation for downlink NOMA systems: Key techniques and open issues," IEEE Wireless Commun., vol. 25, no. 2, pp. 40-47, Apr. 2018.

[20] Z. Ding et al., "Application of non-orthogonal multiple access in LTE and 5G networks," IEEE Commun. Mag., vol. 55, no. 2, pp. 185-191, Feb. 2017.

[21] M. Zeng, et al., "Capacity comparison between MIMO-NOMA and MIMO-OMA with multiple users in a cluster," IEEE J. Sel. Areas Commun., vol. 35, no. 10, pp. 2413-2424, Oct. 2017.

[22] S. Boyd and L. Vandenberghe, Convex Optimization. Cambridge, UK: Cambridge Univ. Press, 2004. 